\title{
Prefrontal Hypoactivity Associated with Impaired Inhibition in Stimulant-Dependent Individuals but Evidence for Hyperactivation in their Unaffected Siblings
}

\author{
Sharon Morein-Zamir*, 1,2, P Simon Jones ${ }^{1,2}$, Edward T Bullmore ${ }^{1,2,3,4}$, Trevor W Robbins ${ }^{1,5}$ and \\ Karen D Ersche ${ }^{1,2}$ \\ 'Behavioural and Clinical Neuroscience Institute, University of Cambridge, Cambridge, UK; ' ${ }^{2}$ Department of Psychiatry, University of Cambridge, \\ Cambridge, UK; ${ }^{3}$ Cambridgeshire and Peterborough NHS Foundation Trust, Cambridge, UK; ${ }^{4}$ Clinical Unit Cambridge, GlaxoSmithKline, \\ Addenbrooke's Centre for Clinical Investigations, Cambridge, UK; ${ }^{5}$ Department of Psychology, University of Cambridge, Cambridge, UK
}

\begin{abstract}
A neurocognitive endophenotype has been proposed for stimulant dependence, based on behavioral measures of inhibitory response control associated with white matter changes in the frontal cortex. This study investigated the functional neuroimaging correlates of inhibitory response control, as functional activity serves as a more dynamic measure than brain structure, allowing refinement of the suggested endophenotype. Stimulant-dependent individuals (SDIs), their unaffected siblings (SIBs), and healthy controls (CTs) performed the stop-signal task, including stop-signal reaction time (SSRT) as a measure of response inhibition, while undergoing functional magnetic resonance imaging. SDIs had impaired response inhibition accompanied by hypoactivation in the ventrolateral prefrontal cortex (PFC). In addition, they demonstrated hypoactivation in the anterior cingulate when failing to stop. In contrast, no hypoactivations were noted in their unaffected SIBs. Rather, they exhibited increased activation in the dorsomedial PFC relative to controls, together with inhibitory performance that was intermediate between that of the stimulant group and the healthy CT group. Such hyperactivations within the neurocircuitry underlying response inhibition and control are suggestive of compensatory mechanisms that could be protective in nature or could reflect coping with a pre-existing vulnerability, thus expressing potential aspects of resilience. The functional activation associated with response inhibition and error monitoring showed differential patterns of results between SDls and their unaffected first-degree relatives, suggesting that the proposed endophenotype does not generalize to functional brain activity.

Neuropsychopharmacology (2013) 38, 1945-1953; doi: I0.1038/npp.2013.90; published online 29 May 2013
\end{abstract}

Keywords: endophenotypes; $\mathrm{fMRl}$; stimulant dependence; stop-signal; cognitive control; drug use

\section{INTRODUCTION}

Difficulties in exerting control over behaviors such as drug seeking are a hallmark of drug addiction. Loss of control leads to persistence in drug taking with larger amounts consumed for longer than intended, despite a desire to quit and despite adverse personal and social consequences (American Psychiatric Association, 2000). Impairments in inhibitory control have been proposed as central to various stages of stimulant dependence, such as increasing susceptibility to initial use, transition to dependence, maintenance, as well as contributing to relapse and difficulties in maintaining abstinence (Goldstein and Volkow, 2011). Response inhibition is particularly informative in investigating control functions as in addition to face validity and objective behavioral indices, its underlying neural

*Correspondence: Dr S Morein-Zamir, Behavioural and Clinical Neuroscience Institute, University of Cambridge, Cambridge CB2 3EB, UK, Tel: + 441223767035 , Fax: + 441223336968 ,

E-mail: sm658@cam.ac.uk

Received 17 January 2013; revised I March 2013; accepted 7 March 2013; accepted article preview online 22 April 2013 substrates likely mediate other self-regulation functions implicated in drug abuse (Tabibnia et al, 2011). Moreover, animal models of impaired response inhibition have proven instructive for addiction (Dalley et al, 2011). Converging evidence from patient lesion, imaging, transcranial magnetic stimulation and electrocorticography provide support for involvement of the pre-supplementary motor area (preSMA) in dorsomedial prefrontal cortex (dmPFC) and right ventrolateral PFC (vlPFC), encompassing the anterior insula and inferior frontal gyrus, in response inhibition (Aron et al, 2003; Floden and Stuss, 2006; Levy and Wagner, 2011; Swann et al, 2012; Swick et al, 2011).

One key challenge is identifying the relative contribution of deficient inhibitory function to different stages of stimulant dependence. Chronic stimulant users have difficulties suppressing prepotent responses, and exhibit slowed stopping compared with controls (Ersche et al, 2011; Fernandez-Serrano et al, 2012; Fillmore and Rush, 2002; Kaufman et al, 2003; Monterosso et al, 2005). However, pre-existing genetic and environment vulnerabilities likely interact with short- and long-term effects of drug use on behavior and the brain to produce these deficits. 
Investigating the underlying traits or endophenotypes that are intermediate between predisposing genes and clinical symptoms may prove useful (Gottesman and Gould, 2003). Endophenotypes are quantifiable traits that are (1) associated with the disorder; (2) heritable; (3) largely state independent; (4) co-segregate with the disorder within families; and (5) overrepresented in non-affected family members relative to the general population. As stimulant dependence is highly heritable (Merikangas and McClair, 2012), this approach can elucidate potential behavioral and associated brain abnormalities independent of drug taking. The role of pre-existing vulnerabilities is poorly understood, but evidence suggests impaired response regulation and control in those with a family history of drug or alcohol dependence (Acheson et al, 2011; Dawes et al, 1997; Tarter et al, 2003). Moreover, weak inhibitory control predicted later illicit drug use in individuals with and without a family history of alcoholism, but more strongly in the former (Nigg et al, 2006). Comparing drug-dependent individuals to their siblings (SIBs), who have not become drug dependent, and both to healthy control participants can further address these issues, as shared impairments would not result from drug exposure. Identifying familial vulnerability markers for drug dependence may further lead to the development of effective, preventive, and therapeutic strategies for individuals at risk.

In accordance with this rationale, inhibitory performance using the stop-signal task (Logan, 1994) has recently been explored as part of a neurocognitive endophenotype for stimulant drug dependence (Ersche et al, 2012b, c). This task requires suppressing already initiated motor responses, enlisting greater inhibitory demands, and less action selection than other action inhibition tasks (Eagle et al, 2008). Stimulant-dependent individuals (SDIs) and their unaffected SIBs demonstrated slowed stopping latencies, and shared white matter abnormalities in the vlPFC in both groups were associated with this slowing (Ersche et al, 2012 b , 2012c). Although some gray matter abnormalities were noted in both groups, no association was noted between gray matter integrity and response inhibition difficulties. These results suggest impaired response inhibition as part of a biomarker for familial risk of drug dependence.

Another important aspect of such a neurocognitive biomarker is neural functioning, which provides an important link between brain-based biological underpinnings of the disorder and behavior. Chronic drug abuse is associated with substantial metabolic PFC changes (Goldstein and Volkow, 2011). In go/no-go tasks, nonabstinent cocaine users demonstrated hypoactivity in no-go trials in the insula and pre-SMA (Hester and Garavan, 2004; Kaufman et al, 2003). Reduced activation in SDI was also reported in the anterior cingulate (ACC; Hester and Garavan, 2004; Kaufman et al, 2003; Li et al, 2008), in accordance with medial PFC hypo-responsiveness during errors and high-conflict situations in addiction, believed to reflect diminished error monitoring (Hester and Garavan, 2004; Nestor et al, 2011). Such results suggest executive control-related hypoactivity is not pervasive throughout the PFC with some psychological and neocortical specificity to drug-related inhibitory dysfunction (Kaufman et al, 2003).

We compared functional activation in SDI, their non-affected SIBs, and healthy CTs while performing a stop-signal task. Brain function could reveal additional manifestations of inhibitory vulnerability relating to the SDI endophenotype, but as function is highly adaptable potential protective factors may also be evident. Similar functional abnormalities between SDI and SIB reflecting predisposing vulnerability factors may be of particular utility for prevention and treatment as these could be amenable to change (Vocci, 2008). We anticipated the possibility of shared impairments between the SDI and SIB, as heritability influences on functional activation relating to executive functions have been found in the vlPFC and ACC (Koten et al, 2009; Matthews et al, 2007). Based on previously published findings (Ersche et al, 2012b, c), we hypothesized impaired response inhibition in the siblingpairs using a modified version of the stop-signal adapted for the MR environment. We further hypothesized hypoactivations in brain regions subserving response inhibition in SDI. Critically, if the SIB demonstrated similar hypoactivations then it would suggest functional difficulties in the motor inhibition system despite no exposure to chronic drug use, thus further characterizing the endophenotype. Alternatively, should the SIB demonstrate hyperactivations or other differences in the relevant neuro-circuitry, this may be indicative of compensatory responses possibly relevant to resilience.

\section{MATERIALS AND METHODS}

\section{Participants}

Recruitment and screening procedures have been described in detail elsewhere (Ersche et al, 2012b, c). Briefly, all participants were aged 18-55 years, with no history of psychotic or neurodevelopmental disorder (including Attention Deficit/Hyperactivity Disorder), neurological illness, or traumatic head injury, and were fluent in English. Sibling pairs shared the same biological parents with one sibling satisfying Diagnostic and Statistical Manual, Fourth Edition, Text Revision (DSM-IV-TR; American Psychiatric Association, 2000) criteria for cocaine or amphetamine dependence, and the other with no history of substance dependence except nicotine. All participants were psychiatrically evaluated using the Structured Clinical Interview for DSM-IV (First et al, 2002) augmented with a semistructured interview to ascertain history of drug use, mental, and physical health, including signs of acute intoxication and withdrawal. Participants were recruited by advertisements and from treatment services in East Anglia, UK. Healthy CTs had no personal or family history of drug or alcohol dependence, and were recruited from the community. Verbal IQ was assessed by the National Adult Reading Test (NART; Nelson, 1982). Drugtaking experiences in CT and SIB were assessed with the Drug Abuse Screening Test (DAST-20; Gavin et al, 1989) and Alcohol Use Disorders Identification Test (AUDIT; Saunders et al, 1993). Compulsive drug taking was assessed in the SDI with the Obsessive-Compulsive Drug Use Score (OCDUS; Franken et al, 2002). The SDI had been using stimulants for several years and reported moderate-to-high compulsive symptoms (see Table 1). Of the SDI, 17 met criteria for dependence on opiates, 9 for alcohol and 1 for cannabis. Urine screen results were positive for all, but one, 
Table I Demographic and Clinical Measures

\begin{tabular}{|c|c|c|c|c|c|c|}
\hline \multirow[t]{2}{*}{ Characteristic } & \multicolumn{2}{|c|}{ Stimulant-dependent individuals } & \multicolumn{2}{|c|}{ Siblings } & \multicolumn{2}{|c|}{ Controls } \\
\hline & Mean & SD & Mean & SD & Mean & SD \\
\hline Age (years) & 34.53 & 7.81 & 33.00 & 8.56 & 31.68 & 8.49 \\
\hline Verbal IQ (NART) & 110.67 & 7.33 & 109.08 & 8.85 & 112.00 & 8.34 \\
\hline Alcohol use (AUDIT) & $|1.4|$ & 10.79 & 3.23 & 3.57 & 3.05 & 2.25 \\
\hline Compulsivity (OCDUS) & 24.53 & 9.71 & & & & \\
\hline Duration of stimulant use (years) & 15.87 & 6.71 & & & & \\
\hline Age of stimulant use onset (years) & 16.59 & 3.04 & & & & \\
\hline
\end{tabular}

Abbreviations: NART, National Adult Reading Test; OCDUS, Stimulant-related Obsessive Compulsive Drug Use Scale; DAST-20, Drug Abuse Screening Test, cut-off score for drug abuse: >5; AUDIT, Alcohol Use Disorders Identification Test, cut-off score for alcohol abuse: $>8$.

SDI and negative for all SIB and CT. The study was approved by the Cambridge Research Ethics Committee (REC08/H0308/310; PI KD Ersche) and before participation, volunteers provided written informed consent. Data from these individuals as part of a larger sample have been published previously (Ersche et al, 2012b, 2012c).

\section{Stop-Signal Task}

Participants viewed the task stimuli via a mirror as they lay in the scanner. On go trials, participants pressed left and right buttons, in response to visual go stimuli (left and right pointing white arrows, $1000 \mathrm{~ms}$ ). On stop trials, the go stimulus was followed by a stop signal (orange arrow pointing upwards, $300 \mathrm{~ms}$ ) and participants had to withhold responding. Following initial pilot studies (see Supplementary Information), the MR task was modified to ensure adequate performance in SDI. There were 48 stop trials and 240 go trials, presented intermixed and counterbalanced with left and right, in a single block. The delay between go and stop stimuli, initially set to $250 \mathrm{~ms}$, was adjusted individually by a tracking algorithm in $50 \mathrm{~ms}$ steps to allow 50\% successful stopping (Logan et al, 1997). If a response was recorded before stop signal onset, it did not appear and the trial was repeated. Inter-trialintervals were randomly jittered between 900 and $1100 \mathrm{~ms}$ (Whelan et al, 2012).

\section{Scanning Acquisition}

Functional imaging data were collected in one run on a Siemens TIM Trio 3-Tesla scanner (Erlangen, Germany) using whole-brain echo planer images (EPIs) with the following parameters: repetition time $(\mathrm{TR})=2000 \mathrm{~ms}$; echo time $(\mathrm{TE})=30 \mathrm{~ms}$; flip angle $=78^{\circ} ; 32$ slices with slice thickness $3 \mathrm{~mm}$ plus $0.75 \mathrm{~mm}$ gap; matrix $=64 \times 64$; field of view $(\mathrm{FOV})=192 \times 192 \mathrm{~mm}$ yielding $3 \times 3 \mathrm{~mm}$ in-plane resolution, and the number of volumes ranged from 278 to 305. T1-weighted scans were acquired for registration purposes (176 slices of $1 \mathrm{~mm}$ thickness, $\mathrm{TR}=2300 \mathrm{~ms}$; $\mathrm{TE}=2.98 \mathrm{~ms}$, $\mathrm{TI}=900 \mathrm{~ms}$, flip angle $\left.=9^{\circ}, \mathrm{FOV}=240 \times 256 \mathrm{~mm}\right)$.

\section{Data Analysis}

Mean go reaction time (RT) and stop-signal RT (SSRT) were calculated for each individual. In addition, percent unsuccessful stopping was computed and unsuccessful stop RT was compared with go RT. SSRT was estimated by subtracting mean stop-signal delay from correct go RT in accordance with the race model (Logan, 1994). It became apparent that a subset of participants did not follow instructions, leading to violation of the race model. Full details regarding inclusion criteria adopted to uphold the race model can be found in the Supplementary Information. Following exclusion of three participants due to poor scanning quality, analyses included $41 \mathrm{CT}, 32 \mathrm{SDI}$, and 39 SIB from 143 participants. Behavioral and demographic data were analyzed using $\chi^{2}$ and analysis of variance (ANOVA) tests, significant group differences are followed by Cohen d's effect size.

Imaging data were processed and analyzed using Statistical Parametric Mapping 8 (SPM, http://www.fil.ion. ucl.ac.uk/spm/). The first five volumes were discarded due to T1-equilibrium effects. Images were realigned and mean EPI image was co-registered to the T1-weighted image, which was segmented and warped to the Montreal Neurological Institute template using new segment and the deformations applied to the EPI volumes, which were resampled to $2 \times 2 \times 2 \mathrm{~mm}^{3}$. Finally, images were smoothed with a Gaussian kernel of a 8-mm FWHM.

First level analyses were performed using the general linear model as implemented in SPM8. Individual design matrixes modeled two events of interest: successful stops and unsuccessful stops by convolving onset times with a canonical hemodynamic response function with temporal and dispersion derivatives (see Supplementary Information for further details). First level contrasts were computed from stops relative to baseline and failed $v s$ successful stops. Go trials occurred frequently, comprising the general linear model baseline. Successful and unsuccessful stops were entered into second-level random-effect analyses, comprising one-sample $t$-tests for each group separately and a between-groups ANOVA. Gender was included as a 
covariate in all second-level analyses. Regions associated with stopping comprised a search area used in the ANOVA (see Supplementary Information). Three anatomical ROIs further examined group differences associated with successful stopping, including the right and left pre-SMA and right anterior Insula/frontal Operculum (aIfO; see Supplementary Information) (Tzourio-Mazoyer et al, 2002). For unsuccessful vs successful stops, the right and left ACC comprised ROIs. All results reported were significant at $P<0.05$ corrected for family-wise error with small volume correction, and peak voxels in Montreal Neurological Institute coordinates. Where significant between-group differences were found, MarsBaR (Brett et al, 2002) was used to compute 8-mm spheres around peak coordinates for each individual, which were then correlated with SST performance measures, and in the SDI group, drug use measures. Results were further examined while covarying for tobacco and alcohol consumption.

\section{RESULTS}

\section{Demographic and Clinical Measures}

As seen in Table 1 , age $(\mathrm{F}<1, P>0.30)$ and verbal IQ $(\mathrm{F}=1.1 .8, P>0.30)$ did not differ between groups, but gender distribution differed between SDI and SIB with the latter being predominantly male $\left(\chi^{2}(2)=19.57, P<0.001\right)$. Importantly, SIB and CT both had low drug-taking experiences and alcohol use as reflected by their low DAST-20 and AUDIT scores.

\section{Behavioral Measures}

As seen in Table 2, there were no significant group differences in go RT $(F(2,109)=1.63, P=0.21)$. There was a group difference in $\operatorname{SSRT}(F(2,109)=3.81, P=.025)$ with SDI slower than CT $(P<0.025, d=0.63$; see Table 2$)$. SIB were intermediate, and did not significantly differ from either CT $(P=0.573, d=0.22)$ or SDI $(P=0.185, d=0.44)$. The results remained when covarying for gender with significantly slowed SSRT in SDI compared with CT and intermediate performance in SIB. With regard to the race model, the percent of unsuccessful stop trials did not differ between groups $(F(2,109)=0.18, P=0.834)$ and indicated the tracking algorithm was successful. All groups demonstrated faster unsuccessful stop than go latencies in keeping with the race model (CT: $F(1,40)=4.57, P=.039$; SDI: $F(1,31)=41.197, P<0.001$;
SIB: $F(1,38)=13.579, P<0.001)$. In addition, there were no significant group differences in go RT standard deviation $(F(2,109)=2.33, P=0.10)$ or slowing on go trials following unsuccessful stops $v s$ successful stops $(F(2,109)=0.71$, $P=0.50)$.

\section{Neuroimaging}

Each group demonstrated activation in regions commonly activated in this task, including the pre-SMA, the vlPFC encompassing the insula, and IFG in addition to the inferior parietal cortex and occipital cortex (see Figure 1).

In the ANOVA, there was a single significant cluster in the vlPFC $\left(P=0.038(30,20,-10)\right.$, cluster extent $\left(K_{\mathrm{E}}\right)=165$, $Z=4.28)$. Direct comparisons indicated $\mathrm{CT}$ had greater activation than SDI $\left(P=0.014(32,20,-10), K_{\mathrm{E}}=203\right.$, $Z=4.48$ ). Similarly, SIB had greater activation than SDI in a single cluster at close proximity $(P=0.040$ $\left.(32,22,-6), K_{\mathrm{E}}=271, Z=4.22\right)$. At this stringent level, there were no differences between CT and SIB. Whole-brain pairwise comparisons at $P<0.001$ uncorrected in the Supplementary Information demonstrate the specificity of these results.

The ROI analyses replicated the above finding (see Figure 2). In the right aIfO, a main effect of group revealed a single cluster $\left(P=0.025(34,20,-10), K_{\mathrm{E}}=38, Z=4.00\right)$, with $\mathrm{CT}$ having greater activation than SDI $(P=0.004$ $\left.(34,20,-10), K_{\mathrm{E}}=60, Z=4.46\right)$ and similarly SIB had greater activation than SDI $\left(P=0.045(34,24,-6), K_{\mathrm{E}}=51\right.$, $Z=3.78)$. Additionally, SIB had greater activation than $\mathrm{CT}$ in the left pre-SMA $\left(P=0.033(-12,8,46), K_{\mathrm{E}}=7\right.$, $Z=3.47)$.

Comparing unsuccessful to successful stops revealed a main effect of group in right ACC $(P=0.001(16,32,18)$, $\left.K_{\mathrm{E}}=25, Z=4.53\right)$, with both CT and SIB having greater activation than SDI $\left(P<0.001(16,32,18), K_{\mathrm{E}}=126\right.$, $Z=5.03$ and $P=0.050(12,28,18), K_{\mathrm{E}}=11, Z=3.37$, respectively). The findings were almost identical when using all stop trials. In none of these comparisons did SDI demonstrate increased activation compared with CT and SIB. Nor did the CT show increased activations compared with SIB. There was a small correlation between aIfO activation and SSRT $(t(110)=2.64, P=.009, r=-0.244$ at $(30,20,-10))$, but no significant correlations with go RT. Finally, there was a correlation between SSRT and duration of use in SDI $(t(30)=2.20, P=.036, r=0.373)$. In sum, the SDI demonstrated vlPFC hypoactivation during stopping and ACC hypoactivation during unsuccessful stopping,

Table 2 Stop-signal Task Performance Measures

\begin{tabular}{|c|c|c|c|c|c|c|c|}
\hline \multirow[t]{2}{*}{ Task measure } & \multicolumn{2}{|c|}{ Stimulant-dependent individuals } & \multicolumn{2}{|c|}{ Siblings } & \multicolumn{2}{|c|}{ Controls } & \\
\hline & Mean & SD & Mean & SD & Mean & SD & \\
\hline Go RT & 411.43 & 71.69 & 434.62 & 69.17 & 407.34 & 74.25 & NS \\
\hline SSRT & 233.96 & 51.66 & 211.46 & 51.08 & 199.44 & 56.59 & $P<0.05$ \\
\hline Percent unsuccessful stopping & 49.57 & 2.44 & 49.24 & 2.62 & 49.30 & 2.22 & NS \\
\hline Go SD & 105.54 & 30.14 & 101.69 & 28.39 & 90.74 & 33.50 & NS \\
\hline Slowing following an unsuccessful stop & 18.47 & 54.98 & 21.38 & 39.56 & 31.17 & 51.24 & NS \\
\hline
\end{tabular}



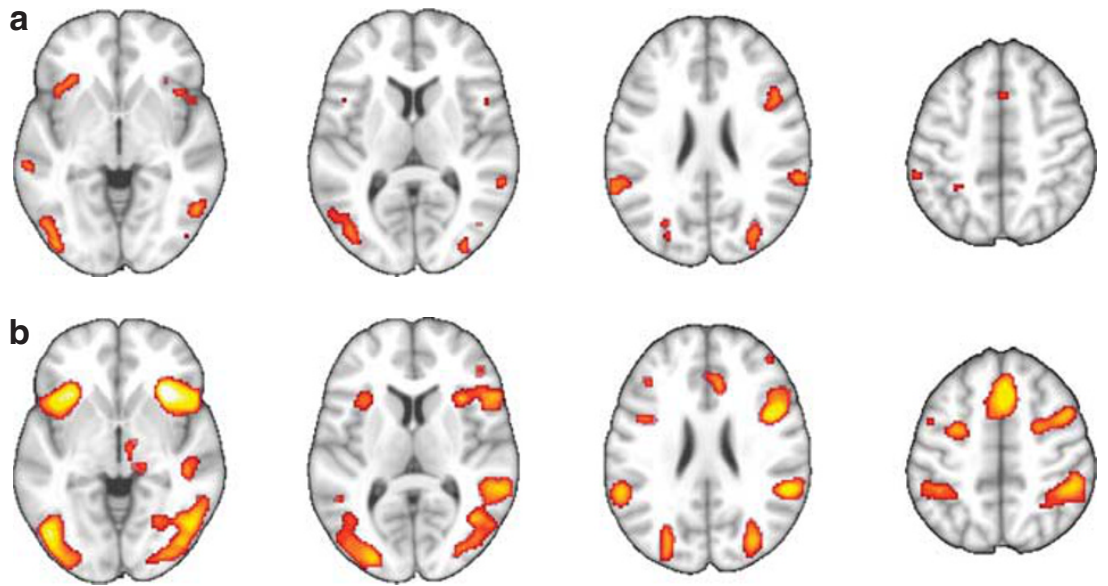

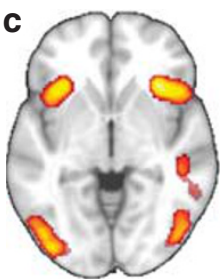

$-4$

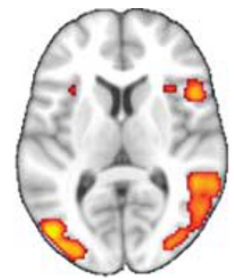

8

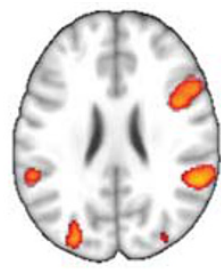

24

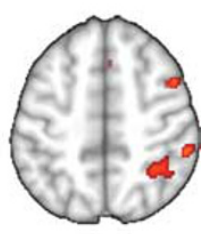

48

Figure I Significant brain activation maps associated with stopping in each group $(P<0.05$, Family-wise error). Note. Brain activation maps per group: row (a) stimulant-dependent individuals; row (b) unaffected siblings of stimulant-dependent individuals; row (c) healthy controls. Axial brain slices demonstrate main activation clusters per group at family-wise error $P<0.05$. The $z$-coordinate slices are: $-4,8,24$ and 48 . Right side of each slice corresponds to the right side of the brain.

compared with the other two groups. The SIB had increased activation in the pre-SMA compared with CT. With the exception of the error-related ACC hypoactivation, the results remained covarying with alcohol and tobacco consumption (see Supplementary Information).

\section{Discussion}

We used functional magnetic resonance imaging (fMRI) to elucidate functional correlates of response inhibition associated with family vulnerability for stimulant dependence. In all groups, stopping was associated with similar widespread activations throughout prefrontal, parietal, and occipital regions, although with smaller activation volumes in SDI (Kaufman et al, 2003). Group differences were not widespread, and were restricted to PFC regions subserving response inhibition (Levy and Wagner, 2011; Swick et al, 2011). Stopping in SDI was impaired and associated with relative hypoactivation in several PFC regions. The SIBs exhibited stopping performance that was intermediate between SDI and CTs together with increased dmPFC activation, suggesting increased effort to achieve comparable, if not worse, performance. The diverging results between SDI and SIBs suggest fMRI activation measures associated with response inhibition are unlikely to contribute to an endophenotype. Potential compensatory hyperactivations within the neurocircuitry-mediating response inhibition in the SIBs point to the possibility that underlying vulnerabilities in executive functions may be overcome in individuals at risk of stimulant drug abuse under certain conditions.

\section{Response Inhibition as an Endophenotype for Stimulant Dependence}

A putative endophenotype would reflect shared abnormalities in family members rendering them more vulnerable to drug dependence. Despite intermediate performance levels, often reported for potential endophenotypes, abnormal functional correlates differed considerably between SIBs and SDI. The SIBs, manifesting increased familial risk in the absence of drug abuse, did not demonstrate any hypoactivations. Rather they recruited key prefrontal areas successfully and their hemodynamic response resembled CTs rather than SDI. These SIBs have previously shown increased self-report impulsivity and compulsivity compared with CTs and share some abnormalities in brain structure with their stimulant-dependent SIBs (Ersche et al, 2012b, 2010). In fact, shared IFG white matter abnormalities between SDI and SIBs were associated with impaired stopping, although no gray matter abnormalities were detected in regions implicated in the current study. It is possible that measures other than BOLD signal could reveal functional abnormalities in the vlPFC. In addition, tasks sensitive to function in non-PFC areas showing gray matter shared familial abnormalities may reveal potential functional endophenotypes in future. Intriguingly, both the unaffected SIBs and recreational cocaine users have increased gray matter volume in the cerebellum (Ersche et al, 2013), which may prove to be protective. As the present study did not reveal abnormal cerebellar activation, such putative protective factors may involve processes other than response inhibition. Although task-related PFC 

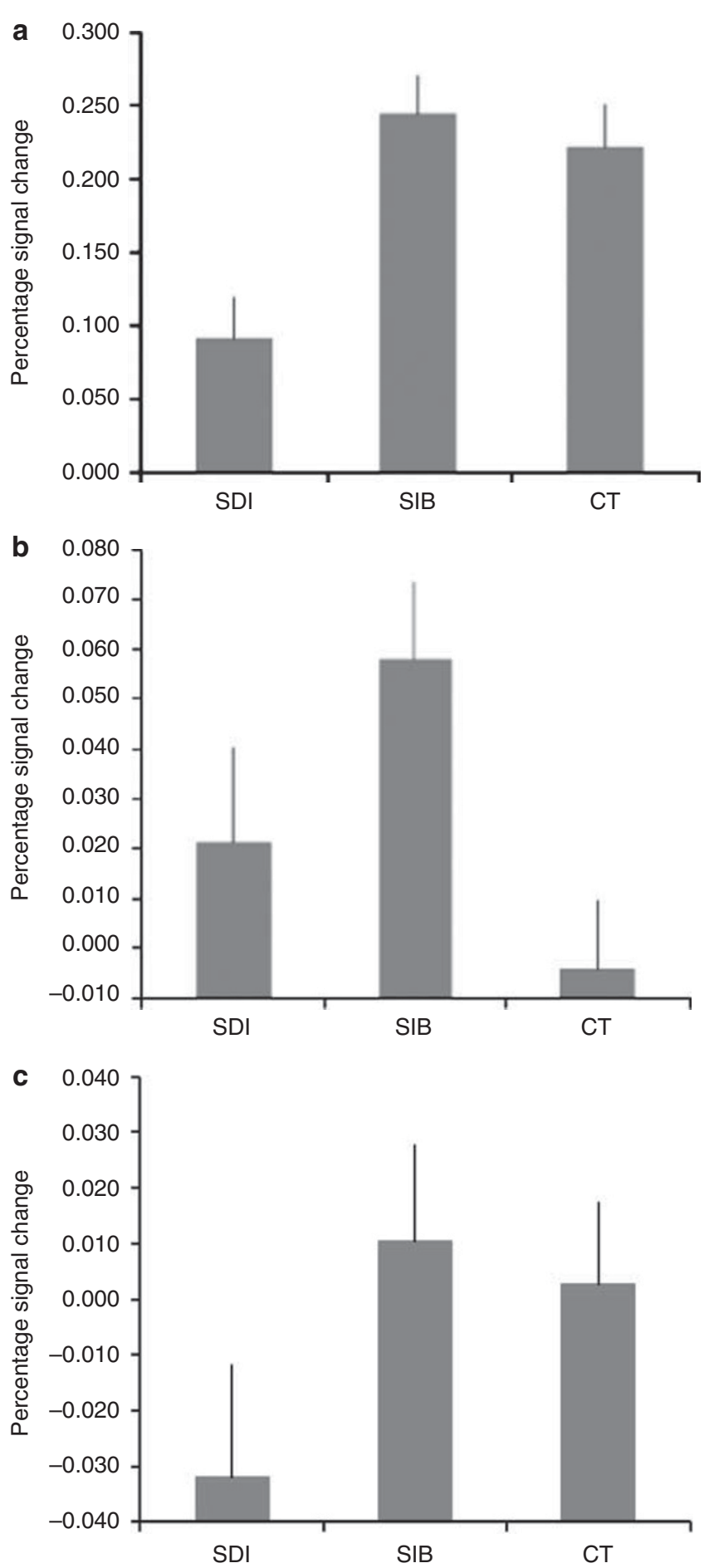

Figure 2 Group mean percent signal change of 8-mm spheres associated with stopping (a and b) and error monitoring (c). (a) Anterioinsula/frontal operculum, centred around coordinates: $x=34, y=20$, $z=-10$; (b) pre-SMA, centred around coordinates: $x=-12, y=8$, $z=46$; (c) anterior cingulate, centred around coordinates: $x=16, y=32$, $z=18$. Error bars index standard error of the mean. CT, controls; SDI, stimulant dependent individuals; SIB, unaffected siblings.

functional activations have shown good group stability (Blokland et al, 2011) and hereditary influences (Koten et al, 2009; Matthews et al, 2007), these are typically smaller in magnitude than those reported for brain

structure (Peper et al, 2007). Taken together, brain structure associated with inhibitory difficulties can be considered a potential endophenotype for stimulant drug dependence, whereas brain function appears to reveal considerably greater levels of plasticity and variability.

\section{Potential for Compensatory Mechanisms in Unaffected SIBs}

Compared with CTs, SIBs demonstrated localized dmPFC hyperactivation, accompanying slightly (albeit not significantly) worse performance. As the dmPFC, particularly the pre-SMA, is considered a key neural substrate of response inhibition (Floden and Stuss, 2006), it is plausible that the hyperactivations are indicative of compensatory mechanisms. Intriguingly, right vlPFC hyperactivations have been found with illicit substance use in adolescents (Whelan et al, 2012) and with long-term abstinence in cocaine users (Connolly et al, 2012). Presumably such functional compensations could develop during initial stages of drug use, disappear with chronic use, but reemerge following protracted abstinence. These groups did not show dmPFC hyperactivations as seen in the unaffected SIBs. This then may be a marker of neural vulnerability seen in the absence of drug use and its resulting toxic effects. Alternatively, it may reflect protective factors enabling resilience, as the SIBs avoided drug dependence despite initial vulnerability.

Consistent with this possibility, the SIBs exhibited less SSRT slowing than previously reported (Ersche et al, 2012b). The present task was adapted to enable SDI to perform in the challenging MR environment. That the unaffected SIBs exhibit only mild impairments in attention and executive functions (Ersche et al, 2012c), may have allowed them to benefit from a more salient and less unpredictable stop signal. Accordingly, better performance was observed in the current sample in the MR task than the non-MR version. Compared with the others, this was most pronounced in the SIB, who still had slower SSRTs than CTs in the non-MR version (see Supplementary Information).

The SIB performed better than anticipated compared with SDI and CTs, demonstrating that under present task demands, any underlying abnormalities in structure and predisposing vulnerability factors do not necessarily translate to abnormal functional activation. That individuals may compensate for underlying vulnerability factors, suggests avenues for exploration of protective factors against addiction such as training in volitional control. Functional activations, together with a wider array of cognitive and personality measures (Acheson et al, 2011; Ersche et al, 2012c), may also offer a means of distinguishing between endophenotypes of behavioral disinhibition across different addictions.

\section{Drug-Related Effects on Response Inhibition}

The SDI exhibited longer SSRTs and altered regional brain activations that could be attributed to a combination of increased impulsivity, compulsivity, and drug-use effects. Poor inhibitory control was accompanied by reduced activation in the anterior insula extending into the IFG 
(Kaufman et al, 2003; Li et al, 2008), a region consistently engaged, albeit not exclusively, in inhibitory tasks (Levy and Wagner, 2011; Swick et al, 2011). From present data, we cannot conclude unequivocally that the hypoactivation resulted from inhibitory impairments per se rather than related executive functions such as performance monitoring (Li et al, 2006), specific task characteristics (Aichert et al, 2012), or increased response control demands (Dodds et al, 2011). Nevertheless, the hypoactivations are unlikely to reflect general task difficulties as behavioral group differences were specific to SSRT. With a refined understanding of vlPFC sub-regions still emerging, future studies should examine its role in drug use more closely.

Efficient executive control requires monitoring for errors or conflicting response plans (Botvinick et al, 2004), and is particularly impaired in drug abuse (Goldstein and Volkow, 2011). The ACC hypoactivation associated with failed stopping in SDI is consistent with previous findings in response inhibition tasks (Kaufman et al, 2003; Li et al, 2008) and error/conflict monitoring tasks (Bolla et al, 2004). ACC hypoactivity has been reported in users of other drugs such as opiates (Forman et al, 2004) and cannabis (Eldreth et al, 2004). As the SIB did not show ACC hypoactivation, this may not be a useful endophenotype for stimulant drug use. Similarly, as there were no group differences in posterror slowing, this marker may be less sensitive in SDI (see also Hester et al (2007)) than in other impulsivity-related disorders such as ADHD (Schachar et al, 2004). Deficient error and conflict monitoring associated with ACC hypoactivation could have a role in drug abuse development and maintenance as well as abstinence failure (Connolly et al, 2012). Thus, inhibitory and monitoring dysfunctions likely interact synergistically contributing to disorder severity, relapse, and abstinence maintenance difficulties.

Enhancing cognitive control over drug use remains a key challenge for effective pharmacological and behavioral treatments. A temporal profile of inhibitory deficits as the disorder progresses may highlight a time window, where treatment to improve control could improve clinical outcome, including pharmaceutical treatment (eg, methylphenidate or atomoxetine) and behavioral techniques (Castells et al, 2007). Prevention may be further facilitated by cognitive training, aimed at strengthening the neural networks mediating inhibitory control and self-regulation (Vocci, 2008).

\section{Strengths and Limitations}

This study employed a considerably larger sample than previous fMRI studies of response inhibition in SDI, which together with rigorous type-I error control allowed us to highlight specific findings. The stop-signal task has welldefined neural correlates and is of theoretical relevance to drug dependence. The SDI and their SIBs have been well characterized (Ersche et al, 2012c) and this study adds an important dimension to the inhibitory endophenotype in addiction. The SDI did not refrain from taking drugs, but were not under acute administration and completed the task successfully. In short-term abstinence, the findings may be more pronounced and accompanied by additional behavioral and hemodynamic abnormalities (Fillmore et al, 2006). Accordingly, improved performance and increased task-related activation in the PFC are observed with cocaine, methylphenidate as well as with stimulant selfadministration (Garavan et al, 2008; Goldstein and Volkow, 2011; Li et al, 2008). Better characterization in acute dosing, non-abstinence, short-, and long-term abstinence could clarify the magnitude of inhibitory dysfunction, although this may be affected by age and duration of use (Ersche et al, 2012a).

Many SDI, as in most previous studies, were polydrug users, with higher levels of tobacco and alcohol consumption. Critically, this is part of the drug dependence phenotype as individuals rarely restrict themselves to a single substance. In fact, such a sample would be unrepresentative and of limited generalizability. Importantly, SDI still exhibited response inhibition deficits when covarying for cigarette and alcohol use (see Supplementary Information). The vlPFC hypoactivation remained, whereas ACC hypoactivation no longer reached significance. Similar analyses in the SIBs still showed dmPFC hyperactivation. This together with evidence that smokers exhibit lower dmPFC activation (de Ruiter et al, 2012), suggests increased smoking in SIB unlikely to account for the differences observed.

In sum, we provide evidence for functional divergence within the neurocircuitry underlying response inhibition and control between SDI and their SIBs with circumscribed PFC dysfunction in SDI, and abnormal overactivations in their SIBs.

\section{ACKNOWLEDGEMENTS}

This work was funded by a Medical Research Council (MRC) research grant to KDE, ETB, and TWR (G0701497) and was conducted within the Behavioural and Clinical Neuroscience Institute, University of Cambridge, UK, which is supported by a joint award from the MRC and the Wellcome Trust; Both KDE and PSJ were supported by the MRC, SM was supported by a Wellcome Trust grant (089589/Z/09/Z) awarded to TWR. We thank all our volunteers, Abigail Turnton for her assistance with data collection, Sanja Abott for task programming, and the Mental Health Research Network for facilitating patient referrals.

\section{DISCLOSURE}

TWR consults for Cambridge Cognition, Lilly, Lundbeck, Merck, Sharpe and Dohme, Shire Pharmaceuticals, Teva and Chempartners and also holds research grants from Lilly, Lundbeck, and GlaxoSmithKline. ETB is employed part-time by GlaxoSmithKline and part-time by the University of Cambridge and is a shareholder of GlaxoSmithKline. The remaining authors declare no conflict of interest.

\section{REFERENCES}

Acheson A, Richard DM, Mathias CW, Dougherty DM (2011). Adults with a family history of alcohol related problems are more impulsive on measures of response initiation and response inhibition. Drug Alcohol Depend 117: 198-203. 
Aichert DS, Wostmann NM, Costa A, Macare C, Wenig JR, Moller HJ et al (2012). Associations between trait impulsivity and prepotent response inhibition. J Clin Exp Neuropsychol 34: 1016-1032.

American Psychiatric Association (2000). Diagnostic and Statistical Manual of Mental Disorders. IV-Text Revision edn American Psychiatric Association: Washington, DC.

Aron AR, Fletcher PC, Bullmore ET, Sahakian BJ, Robbins TW (2003). Stop-signal inhibition disrupted by damage to right inferior frontal gyrus in humans. Nat Neurosci 6: 115-116.

Blokland GA, McMahon KL, Thompson PM, Martin NG, de Zubicaray GI, Wright MJ (2011). Heritability of working memory brain activation. J Neurosci 31: 10882-10890.

Bolla K, Ernst M, Kiehl K, Mouratidis M, Eldreth D, Contoreggi C et al (2004). Prefrontal cortical dysfunction in abstinent cocaine abusers. J Neuropsychiatry Clin Neurosci 16: 456-464.

Botvinick MM, Cohen JD, Carter CS (2004). Conflict monitoring and anterior cingulate cortex: an update. Trends Cogn Sci 8: 539-546.

Brett M, Anton J, Valabregue R, Poline JB (2002, Region of interest analysis using an SPM toolbox. In: Abstract presented at the 8th International Conference on Functional Mapping of the Human Brain. 2-6 June, Sendai, Japan.

Castells X, Casas M, Vidal X, Bosch R, Roncero C, Ramos-Quiroga JA et al (2007). Efficacy of central nervous system stimulant treatment for cocaine dependence: a systematic review and meta-analysis of randomized controlled clinical trials. Addiction 102: 1871-1887.

Connolly CG, Foxe JJ, Nierenberg J, Shpaner M, Garavan H (2012). The neurobiology of cognitive control in successful cocaine abstinence. Drug Alcohol Depend 121: 45-53.

Dalley JW, Everitt BJ, Robbins TW (2011). Impulsivity, compulsivity, and top-down cognitive control. Neuron 69: 680-694.

Dawes MA, Tarter RE, Kirisci L (1997). Behavioral self-regulation: correlates and 2 year follow-ups for boys at risk for substance abuse. Drug Alcohol Depend 45: 165-176.

de Ruiter MB, Oosterlaan J, Veltman DJ, van den Brink W, Goudriaan AE (2012). Similar hyporesponsiveness of the dorsomedial prefrontal cortex in problem gamblers and heavy smokers during an inhibitory control task. Drug Alcohol Depend 121: 81-89.

Dodds CM, Morein-Zamir S, Robbins TW (2011). Dissociating inhibition, attention, and response control in the frontoparietal network using functional magnetic resonance imaging. Cerebral Cortex 21: 1155-1165.

Eagle DM, Bari A, Robbins TW (2008). The neuropsychopharmacology of action inhibition: cross-species translation of the stopsignal and go/no-go tasks. Psychopharmacology 199: 439-456.

Eldreth DA, Matochik JA, Cadet JL, Bolla KI (2004). Abnormal brain activity in prefrontal brain regions in abstinent marijuana users. Neuroimage 23: 914-920.

Ersche KD, Barnes A, Jones PS, Morein-Zamir S, Robbins TW, Bullmore ET (2011). Abnormal structure of frontostriatal brain systems is associated with aspects of impulsivity and compulsivity in cocaine dependence. Brain 134(Pt 7): 2013-2024.

Ersche KD, Jones PS, Williams GB, Robbins TW, Bullmore ET (2012a). Cocaine dependence: a fast-track for brain ageing? Mol Psychiatry.

Ersche KD, Jones PS, Williams GB, Smith DG, Bullmore ET, Robbins TW (2013). Distinctive personality traits and neural correlates associated with stimulant drug use versus familial risk of stimulant dependence. Biol Psychiatry (e-pub ahead of print).

Ersche KD, Jones PS, Williams GB, Turton AJ, Robbins TW, Bullmore ET (2012b). Abnormal brain structure implicated in stimulant drug addiction. Science 335: 601-604.

Ersche KD, Turton AJ, Chamberlain SR, Muller U, Bullmore ET, Robbins TW (2012c). Cognitive dysfunction and anxiousimpulsive personality traits are endophenotypes for drug dependence. Am J Psychiatry 169: 926-936.
Ersche KD, Turton AJ, Pradhan S, Bullmore ET, Robbins TW (2010). Drug addiction endophenotypes: impulsive versus sensation-seeking personality traits. Biol Psychiatry 68: 770-773.

Fernandez-Serrano MJ, Perales JC, Moreno-Lopez L, Perez-Garcia M, Verdejo-Garcia A (2012). Neuropsychological profiling of impulsivity and compulsivity in cocaine dependent individuals. Psychopharmacology (Berl) 219: 673-683.

Fillmore MT, Rush CR (2002). Impaired inhibitory control of behavior in chronic cocaine users. Drug Alcohol Depend 66: 265-273.

Fillmore MT, Rush CR, Hays L (2006). Acute effects of cocaine in two models of inhibitory control: implications of non-linear dose effects. Addiction 101: 1323-1332.

First MB, Spitzer RL, Gibbon M, Williams JBW (2002). Structured Clinical Interview for DSM-IV-TR Axis I Disorders, Research Version, Non-patient Edition. (SCID-I/NP) edn. Biometrics Research, New York State Psychiatric Institute: New York.

Floden D, Stuss DT (2006). Inhibitory control is slowed in patients with right superior medial frontal damage. J Cogn Neurosci 18: $1843-1849$

Forman SD, Dougherty GG, Casey BJ, Siegle GJ, Braver TS, Barch DM et al (2004). Opiate addicts lack error-dependent activation of rostral anterior cingulate. Biol Psychiatry 55: 531-537.

Franken IHA, Hendriks VM, Van den Brink W (2002). The obsessive compulsive drug use scale and the desires for drug questionnaire. Addict Behav 27: 675-685.

Garavan H, Kaufman JN, Hester R (2008). Acute effects of cocaine on the neurobiology of cognitive control. Philos Trans $R$ Soc Lond B Biol Sci 363: 3267-3276.

Gavin DR, Ross HE, Skinner HA (1989). Diagnostic validity of the drug abuse screening test in the assessment of DSM-III drug disorders. Br J Addict 84: 301-307.

Goldstein RZ, Volkow ND (2011). Dysfunction of the prefrontal cortex in addiction: neuroimaging findings and clinical implications. Nat Rev Neurosci 12: 652-669.

Gottesman II, Gould TD (2003). The endophenotype concept in psychiatry: etymology and strategic intentions. Am J Psychiatry 160: 636-645.

Hester R, Garavan H (2004). Executive dysfunction in cocaine addiction: evidence for discordant frontal, cingulate, and cerebellar activity. J Neurosci 24: 11017-11022.

Hester R, Simoes-Franklin C, Garavan H (2007). Post-error behavior in active cocaine users: poor awareness of errors in the presence of intact performance adjustments. Neuropsychopharmacology 32: 1974-1984.

Kaufman JN, Ross TJ, Stein EA, Garavan H (2003). Cingulate hypoactivity in cocaine users during a GO-NOGO task as revealed by event-related functional magnetic resonance imaging. J Neurosci 23: 7839-7843.

Koten JW Jr, Wood G, Hagoort P, Goebel R, Propping P, Willmes $\mathrm{K}$ et al (2009). Genetic contribution to variation in cognitive function: an FMRI study in twins. Science 323: 1737-1740.

Levy BJ, Wagner AD (2011). Cognitive control and right ventrolateral prefrontal cortex: reflexive reorienting, motor inhibition, and action updating. Ann N Y Acad Sci 1224: 40-62.

Li CS, Huang C, Yan P, Bhagwagar Z, Milivojevic V, Sinha R (2008). Neural correlates of impulse control during stop signal inhibition in cocaine-dependent men. Neuropsychopharmacology 33: 1798-1806.

Li CS, Milivojevic V, Kemp K, Hong K, Sinha R (2006). Performance monitoring and stop signal inhibition in abstinent patients with cocaine dependence. Drug Alcohol Depend 85: 205-212.

Logan GD (1994). On the ability to inhibit thought and action: A users' guide to the stop signal paradigm. In: Dagenbach DCarr $\mathrm{TH}$ (eds). Inhibitory Processes in Attention, Memory, and Language. Academic Press: San Diego, pp 214-249.

Logan GD, Schachar RJ, Tannock R (1997). Impulsitiry and inhibitory control. Psychol Sci 8: 60-64. 
Matthews SC, Simmons AN, Strigo I, Jang K, Stein MB, Paulus MP (2007). Heritability of anterior cingulate response to conflict: an fMRI study in female twins. NeuroImage 38: 223-227.

Merikangas KR, McClair VL (2012). Epidemiology of substance use disorders. Hum Genet 131: 779-789.

Monterosso JR, Aron AR, Cordova X, Xu J, London ED (2005). Deficits in response inhibition associated with chronic methamphetamine abuse. Drug Alcohol Depend 79: 273-277.

Nelson HE (1982). The National Adult Reading Test Manual. NFER-Nelson: Windsor.

Nestor LJ, Ghahremani DG, Monterosso J, London ED (2011). Prefrontal hypoactivation during cognitive control in early abstinent methamphetamine-dependent subjects. Psychiatry Res 194: 287-295.

Nigg JT, Wong MM, Martel MM, Jester JM, Puttler LI, Glass JM et al (2006). Poor response inhibition as a predictor of problem drinking and illicit drug use in adolescents at risk for alcoholism and other substance use disorders. J Am Acad Child Adolesc Psychiatry 45: 468-475.

Peper JS, Brouwer RM, Boomsma DI, Kahn RS, Hulshoff Pol HE (2007). Genetic influences on human brain structure: a review of brain imaging studies in twins. Hum Brain Mapp 28: 464-473.

Saunders JB, Aasland OG, Babor TF, de la Fuente JR, Grant M (1993). Development of the Alcohol Use Disorders Identification Test (AUDIT): WHO Collaborative Project on Early Detection of Persons with Harmful Alcohol Consumption-II. Addiction 88: 791-804.

Schachar RJ, Chen S, Logan GD, Ornstein TJ, Crosbie J, Ickowicz A et al (2004). Evidence for an error monitoring deficit in attention deficit hyperactivity disorder. J Abnorm Child Psychol 32: 285-293.

Swann NC, Cai W, Conner CR, Pieters TA, Claffey MP, George JS et al (2012). Roles for the pre-supplementary motor area and the right inferior frontal gyrus in stopping action: electrophysiological responses and functional and structural connectivity. Neuroimage 59: 2860-2870.

Swick D, Ashley V, Turken U (2011). Are the neural correlates of stopping and not going identical? Quantitative meta-analysis of two response inhibition tasks. Neuroimage 56: 1655-1665.

Tabibnia G, Monterosso JR, Baicy K, Aron AR, Poldrack RA, Chakrapani S et al (2011). Different forms of self-control share a neurocognitive substrate. J Neurosc 31: 4805-4810.

Tarter RE, Kirisci L, Mezzich A, Cornelius JR, Pajer K, Vanyukov $M$ et al (2003). Neurobehavioral disinhibition in childhood predicts early age at onset of substance use disorder. Am J Psychiatry 160: 1078-1085.

Tzourio-Mazoyer N, Landeau B, Papathanassiou D, Crivello F, Etard O, Delcroix N et al (2002). Automated anatomical labeling of activations in SPM using a macroscopic anatomical parcellation of the MNI MRI single-subject brain. Neuroimage 15: 273-289.

Vocci FJ (2008). Cognitive remediation in the treatment of stimulant abuse disorders: a research agenda. Exp Clin Psychopharmacol 16: 484-497.

Whelan R, Conrod PJ, Poline JB, Lourdusamy A, Banaschewski T, Barker GJ et al (2012). Adolescent impulsivity phenotypes characterized by distinct brain networks. Nat Neurosci 15: $920-925$.

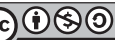

This work is licensed under a Creative Commons Attribution-NonCommercial-ShareAlike 3.0 Unported License. To view a copy of this license, visit http:// creativecommons.org/licenses/by-nc-sa/3.0/

Supplementary Information accompanies the paper on the Neuropsychopharmacology website (http://www.nature.com/npp) 